\title{
Long-term results of the frozen elephant trunk technique for the extensive arteriosclerotic aneurysm
}

\author{
Naomichi Uchida, MD, ${ }^{\mathrm{a}}$ Hidenori Shibamura, MD, ${ }^{\mathrm{a}}$ Akira Katayama, MD, ${ }^{\text {a }}$ Miwa Sutoh, MD, ${ }^{\mathrm{a}}$ \\ Masatsugu Kuraoka, MD, ${ }^{\mathrm{a}}$ and Hiroshi Ishihara, $\mathrm{MD}^{\mathrm{b}}$
}

Objectives: The objective of this report is to elucidate the feasibility of the frozen elephant trunk technique as a one-stage operation for extensive arteriosclerotic aneurysms and to investigate the long-term durability and efficacy of this procedure from our 11 years of experience.

\begin{abstract}
Methods: The subjects were 58 consecutive patients who electively received the frozen elephant trunk technique for arteriosclerotic aneurysms involving the aortic arch and the descending aorta between September 1997 and September 2008. Concomitant procedures included 15 coronary artery bypass grafts, 2 aortic valve replacements, 1 aortic root replacement, and 3 maze procedures. The stent graft was delivered to the seventh thoracic vertebra level (Th7) in 22, Th8 in 26, Th9 in 8, and Th10 in 2 patients. Cerebrospinal fluid drainage was administered preoperatively in $10(17.2 \%)$ patients.
\end{abstract}

Results: Operative mortality within 30 days was 0 of 58 . There was 1 in-hospital death. Perioperative morbidity included strokes and spinal cord injury in $2(3.4 \%)$ patients each. During the mean follow-up period of $54.2 \pm 36.9$ months, there were $9(15.5 \%)$ late deaths, and $7(12.1 \%)$ patients required additional intervention. The 8-year survival was $65.5 \%$, the overall 8 -year aortic event free survival was $72.8 \%$, and the 8 -year event free survival on the site of the stent graft was $94.8 \%$. A follow-up computed tomographic image was available for $86.2 \%$ (50/58) of patients who survived longer than 6 months. The size of the aneurysm increased in $1(2.0 \%)$ patient, was not changed in $6(12.0 \%)$ patients, shrank in $34(68.0 \%)$ patients, and was obliterated in $9(18.0 \%)$ patients.

Conclusions: The frozen elephant trunk for extensive aortic aneurysms had long-term durability and efficacy and might become the alternative treatment for extended aortic replacement. (J Thorac Cardiovasc Surg 2010;139:913-7)

Patients with extensive aortic aneurysms involving the ascending aorta, aortic arch, and the descending aorta are still considered to be a challenge for many cardiovascular surgeons. Operative methods to accomplish extensive aortic replacement have been devised, such as a one-stage approach, ${ }^{1,2}$ a two-stage approach, ${ }^{3-5}$ and hybrid treatments using a stent graft. ${ }^{6-12}$ Another operation that has been introduced is the frozen elephant trunk technique. ${ }^{6-10}$ This operation is performed through a median sternotomy, and an endovascular stent graft is placed into the descending aorta in an antegrade fashion through the opened aortic arch. Then, the ascending aorta and the aortic arch are replaced conventionally. This technique has a combination of advantages of surgical and interventional approaches and, therefore, appears to be promising. The objective of this report is to elucidate the feasibility of this technique for extensive arteriosclerotic aneurysms involving the aortic arch and

\footnotetext{
From the Division of Cardiovascular Surgery, Hiroshima-city Asa General Hospital, ${ }^{\text {a }}$ and Kajikawa Private Hospital, ${ }^{\text {b }}$ Hiroshima, Japan.

Disclosures: None.

Received for publication Nov 25, 2008; revisions received May 9, 2009; accepted for publication Aug 10, 2009; available ahead of print Nov 12, 2009.

Address for reprints: Naomichi Uchida, MD, Division of Cardiovascular Surgery,

Hiroshima-city Asa General Hospital, 2-1-1, Kabe-minami, Asa-Kita-Ku, Hirosh-

ima, Japan, 731-0293 (E-mail: uchidacvs@yahoo.co.jp).

$0022-5223 / \$ 36.00$

Copyright (C) 2010 by The American Association for Thoracic Surgery

doi:10.1016/j.jtcvs.2009.08.018
}

the descending aorta and to investigate the long-term durability and efficacy of this hybrid procedure from our 11 years of experience.

\section{PATIENTS AND METHODS \\ Patients}

The use of endovascular stent grafts was approved by the Ethical Committee in Hiroshima City Asa General Hospital in 1987. The retrospective review of the records was approved by the institutional review board in 2008.

Informed consent was required in each case. We electively performed the frozen elephant trunk technique for extensive arteriosclerotic aneurysms involving the aortic arch and the descending aorta in 58 consecutive patients between September 1997 and September 2008. All operations were done by 2 surgeons (Ishihara and Uchida). The patients consisted of 52 men and 6 women aged from 57 to 84 years, with a mean age of 73.9 years; 29 $(50 \%)$ patients were older than 75 years. Preoperative patient profiles are summarized in Table 1.

\section{Operative Indications}

Operative indications were limited to patients who were judged by two surgeons to have a relatively high operative risk: (1) distal arch aneurysms unable to be treated by thoracic endovascular aortic repair involving the left subclavian artery and (2) difficulty in using a median approach extending to the upper middle descending aorta to perform distal anastomosis. The diameter of the distal normal edge was less than $35 \mathrm{~mm}$ on preoperative computed tomography (CT). A self-expanding Z-shaped stent with the tip 5 $\mathrm{cm}$ on the distal side was attached to a polyester fabric prosthesis (UBE graft; UBE Industries Ltd, Tokyo, Japan) with a diameter between 24 and $37.5 \mathrm{~mm}$ using 5-0 monofilament sutures. These stent grafts were prepared 


\section{Abbreviations and Acronyms \\ $\mathrm{CT}=$ computed tomography \\ $\mathrm{Th}=$ thoracic vertebra}

preoperatively for use. The stent graft was set at $110 \%$ of the diameter of the distal descending aorta and $5 \mathrm{~cm}$ longer than the distance from the distal normal edge on preoperative 3-dimensional CT scans as verified by intraoperative transesophageal echocardiography.

\section{Operative Procedures}

The frozen elephant trunk technique has been described in previous reports. ${ }^{9,13}$ After the rectal temperature had decreased to $28^{\circ} \mathrm{C}$, the aortic arch was incised longitudinally until immediately before the origin of the left subclavian artery via a median sternotomy with arterial perfusion from the right axillary artery. At the end point, the aortic arch was dissected transversely and the stent graft was placed through a $30 \mathrm{~F}$ introducer, which was inserted into the descending aorta with selective cerebral perfusion. The stent graft delivery was technically successful in all 58 patients. The earlier 22 patients (group 1; until July 2002) underwent left subclavian bypass from the ascending bypass except for patients with dilatation of the ascending aorta. The latter 36 patients (group 2; after August 2002) routinely underwent ascending and total arch replacement.

Concomitant procedures included 15 coronary artery bypass grafts, 2 aortic valve replacements, 1 aortic root replacement, and 3 maze procedures for chronic atrial fibrillation. The average operative, cardiopulmonary bypass, selective cerebral perfusion, and circulatory arrest times of the lower body were $342,148,72$, and 35 minutes, respectively. The stent graft was delivered to the seventh thoracic vertebra level (Th7) in 22, Th8 in 26, Th9 in 8 , and Th10 in 2 patients (Table 2).

\section{Patient Follow-up}

Follow-up with CT imaging was obtained 1 month and 6 months after the operation and yearly thereafter. A follow-up CT image was available for $86.2 \%(50 / 58)$ of patients who survived longer than 6 months. Follow-up clinical status was obtained by medical records from an outpatient clinic. Follow-up was $100 \%$ complete, averaging $54.2 \pm 36.9$ months (maximal follow-up 135 months; 25 patients remaining at risk at 5 years). The postoperative change of the aneurysm was classified as showing increase, no change, shrinkage, or obliteration. Shrinkage was defined as a decrease in the size of the aneurysm of more than $3 \mathrm{~mm}$. Obliteration was defined as thrombus from the stent graft becoming less than $3 \mathrm{~mm}$ thick.

\section{Statistical Analysis}

All data were reviewed retrospectively. Continuous data were expressed as means \pm standard deviations. Categorical data were expressed as counts and proportions. Survival, freedom from overall aortic event including an endoleak, and freedom from events on the site of the stent graft were estimated by the Kaplan-Meier method.

\section{RESULTS \\ Early Results}

Operative mortality within 30 days was $0(0 \%)$ of 58 . There was 1 in-hospital death. This patient, who had stenting to the left main bronchial lesion owing to atelectasis resulting from compression by an enormous aneurysm 3 months after the operation, suddenly died of aortoesophageal fistula 7 months after undergoing the frozen elephant trunk technique. Perioperative morbidity included strokes in 2
TABLE 1. Patient characteristics

\begin{tabular}{lc}
\hline No. & 58 \\
Male & 52 \\
Age, y (mean) & 73.9 \\
$>75$ y (\%) & $29(50.0)$ \\
Aneurysm diameter, cm (mean \pm SD) & $6.3 \pm 1.3$ \\
Type of aneurysm, n (\%) & \\
Saccular & $19(32.8)$ \\
Fusiform & $39(67.2)$ \\
Preoperative comorbidities, n (\%) & \\
Old cerebral infarction & $16(27.6)$ \\
Coronary artery disease & $21(36.2)$ \\
Atrial fibrilation & $7(12.1)$ \\
Chronic obstructive pilmonary disease & $17(28.6)$ \\
Renal dysfunction (creatine $>1.5$ mg/dL) & $13(22.4)$ \\
Hypertension & $53(91.2)$ \\
Diabetes & $15(25.9)$ \\
Previous operation, n (\%) & \\
Coronary artery bypass & $4(6.9)$ \\
Descending aorta & $2(3.4)$ \\
Abdominal aorta & $7(12.1)$ \\
Cancer & $4(6.9)$ \\
\hline
\end{tabular}

$(3.4 \%)$ cases, spinal cord injury in $2(3.4 \%)$, pneumonia in $5(8.6 \%)$, re-exploration for bleeding in $1(1.7 \%)$, and renal failure in $1(1.7 \%)$ (Table 3$)$. One of the 2 patients from group 1 with spinal cord injury (treated in 1998), who was the third patient to undergo the frozen elephant trunk technique, had complete paraplegia because the stent graft was distally positioned on the Th9 level without perfusion through the left subclavian artery. On the basis of this experience, we routinely used selective cerebral perfusion through three neck vessels including the left subclavian artery starting with the fourth case. A patient from group 2, who was the 21st patient to undergo the frozen elephant trunk technique (treated in 2002), had paraparesis because he had had previous surgery of an abdominal aortic aneurysm. After this experience, cerebrospinal fluid drainage was performed the day before the operation in elective

\section{TABLE 2. Operative data}

\begin{tabular}{lc}
\hline Surgical time (min) \pm SD & $342 \pm 81$ \\
Cardiopulmonary bypass time (min) \pm SD & $148 \pm 34$ \\
Selective cerebral perfusion time (min) \pm SD & $72 \pm 12$ \\
Open distal anastomosis time (min) \pm SD & $35 \pm 9$ \\
Concomitant operation $(\%)$ & \\
Coronary artery bypass & $15(25.9)$ \\
Aortic valve replacement & $2(3.4)$ \\
Aortic root replacement & $1(1.7)$ \\
Maze procedure & $3(5.2)$ \\
Distal landing zone $(\%)$ & \\
Th7 & $22(37.9)$ \\
Th8 & $26(44.8)$ \\
Th9 & $8(13.8)$ \\
Th10 & $2(3.4)$ \\
\hline
\end{tabular}

$T h$, Thoracic vertebrae. 
TABLE 3. Postoperative data

\begin{tabular}{lc}
\hline Early mortality, $\mathrm{n}(\%)$ & 0 \\
Operative death & $1(1.7)$ \\
In-hospital death & \\
Early morbidities, $\mathrm{n}(\%)$ & $2(3.4)$ \\
Stroke & $2(3.4)$ \\
Spinal cord injury & $1(1.7)$ \\
Re-exploration for bleeding & 0 \\
Mediastinitis & $5(8.6)$ \\
Pneumonia & $1(1.7)$ \\
Renal failure & $54.2 \pm 36.9$ \\
Follow-up period (mo) \pm SD & $9(15.5)$ \\
Late mortality, $\mathrm{n}(\%)$ & 1 \\
Acute type A dissection & 2 \\
Heart failure & 1 \\
Stroke & 2 \\
Pneumonia & 3 \\
Cancer & $7(12.1)$ \\
Additional operation, $\mathrm{n}(\%)$ & 1 \\
Bronchial stenting for atelectasis & 1 \\
Ascending aortic replacement & 1 \\
Aortic root replacement & 1 \\
Thoracoabdominal aortic replacement & 3 \\
Abdominal aortic replacement & \\
\hline
\end{tabular}

patients with a history of aortic repair in the thoracoabdominal or abdominal aorta or when the pathologic condition required stent graft delivery lower than Th9 from January 2002. Cerebrospinal fluid drainage was administered preoperatively in $10(17.2 \%)$ patients.

\section{Late Results}

During the mean follow-up period of $54.2 \pm 36.9$ months, there were $9(15.5 \%)$ late deaths caused by acute type A dissection ( $\mathrm{n}=1$, in-hospital death 6 months after the dissection operation) and nonaortic events $(\mathrm{n}=8)$ such as heart failure $(\mathrm{n}=2)$, stroke $(\mathrm{n}=1)$, pneumonia $(\mathrm{n}=2)$, and cancer $(\mathrm{n}=3)$. The survival was $65.5 \%$ at 8 years (Figure 1$)$. The patient with acute type A dissection who had undergone reconstruction from the ascending aorta to the left subclavian artery without ascending and total arch replacement during the primary frozen elephant trunk procedure had a new intimal tear at the ascending aorta 64 months after the elephant trunk procedure that was not associated with the previous anastomosis. Seven $(12.1 \%)$ patients required additional intervention: bronchial stenting for atelectasis in group 1 resulting from compression by an enormous aneurysm 3 months after operation $(\mathrm{n}=1)$, ascending aortic replacement for acute type A dissection $(\mathrm{n}=1)$, aortic root replacement for root dilatation $(\mathrm{n}=1)$, thoracoabdominal replacement $(\mathrm{n}=1)$, and abdominal aortic replacement $(\mathrm{n}=3)$ (Table 3). Freedom from an overall aortic event including an endoleak was $72.8 \%$ at 8 years (Figure 2). Stent graft-related events were bronchial stenting for atelectasis and thoracoabdominal replacement, and the rate of freedom

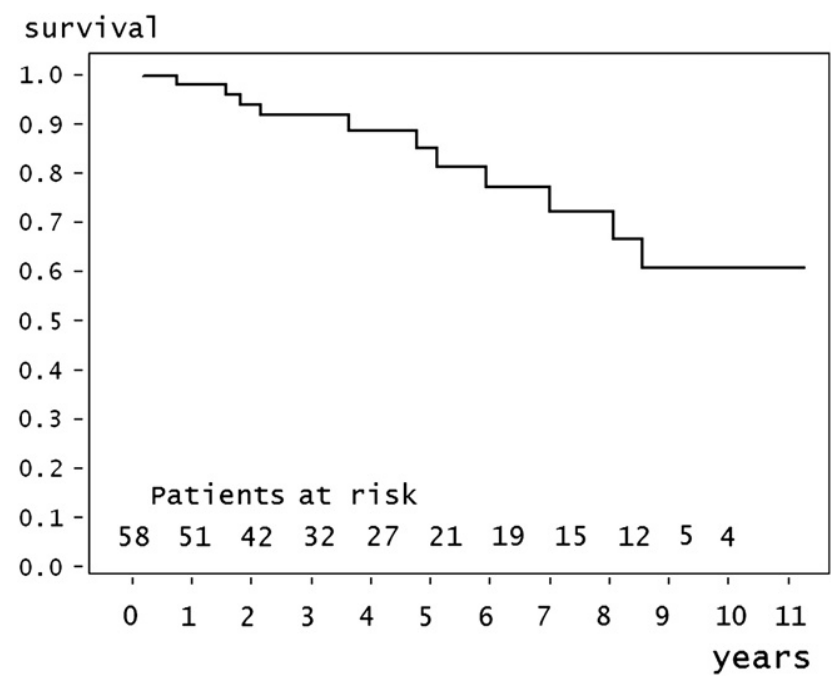

FIGURE 1. The 8-year survival was $65.5 \%$.

from events at the site of stent graft was $94.8 \%$ at 8 years (Figure 3).

A follow-up CT image was available for $86.2 \%(50 / 58)$ of patients who survived longer than 6 months. The size of the aneurysm increased in $1(2.0 \%)$ patient, was not changed in $6(12.0 \%)$ patients, shrank in $34(68.0 \%)$ patients, and was obliterated in $9(18.0 \%)$ patients (Table 4$)$. In a patient whose aortic aneurysm increased in size, a distal endoleak occurred 42 months after the operation, and he was listed for elective thoracic endovascular aortic repair.

\section{DISCUSSION}

It has been reported that the elephant trunk technique with a distal stent graft (frozen elephant trunk) is less invasive and achieves good midterm results. ${ }^{6-10,13,14}$ The frozen elephant trunk technique obtains an anastomosis on the distal

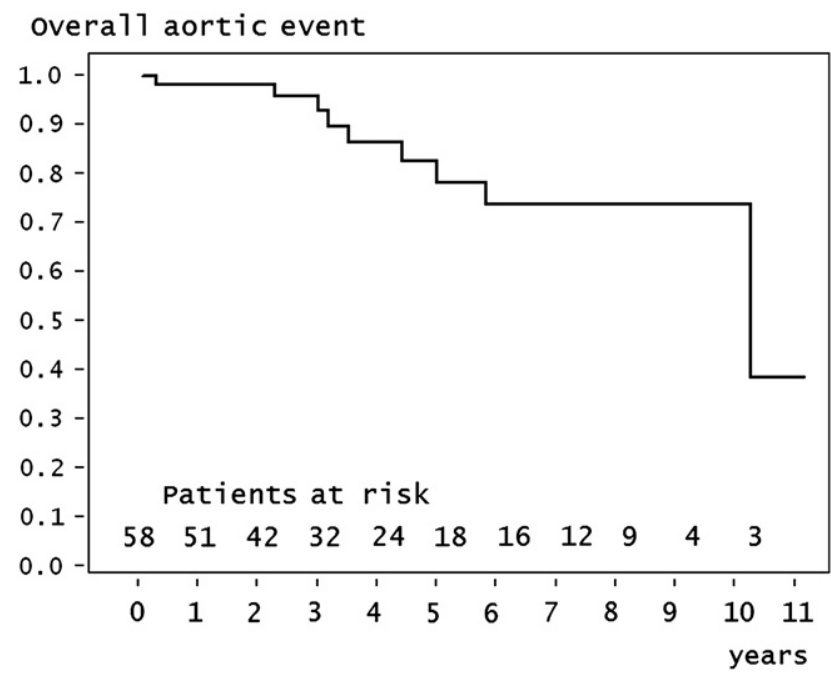

FIGURE 2. The overall 8-year event free rate was $72.8 \%$. 
aortic event (stent graft)

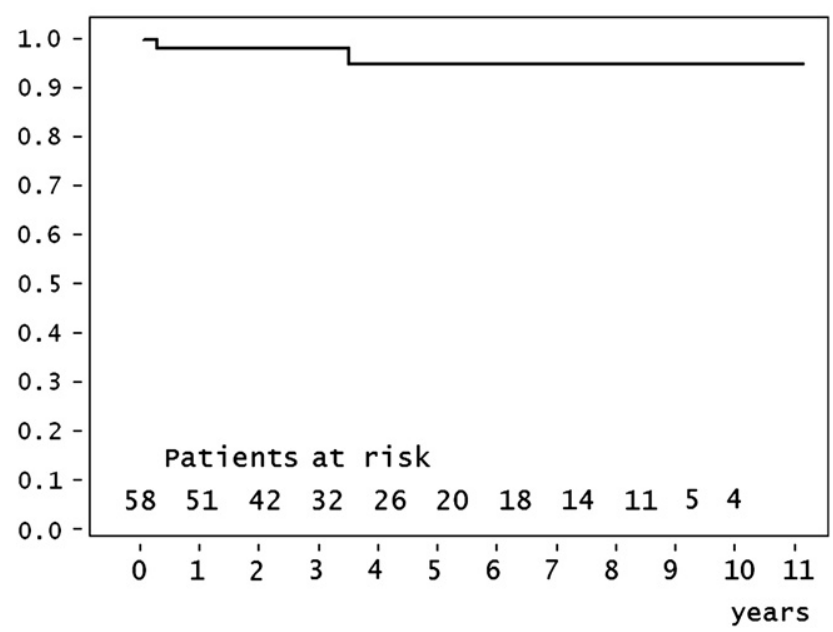

FIGURE 3. The 8 -year event free rate on the site of stent graft was $94.8 \%$.

anastomotic portion by substituting the expansile force of the stented graft for the suture and on the proximal anastomotic portion by suture, where it is hard to adapt the stent anastomosis. Therefore, the frozen elephant trunk can respond to anatomic limitations on the proximal neck vessel as a disadvantage of stent graft technique and anastomotic difficulties on the distal portion. In particular, the frozen elephant trunk technique can be used for extended thoracic repair beyond the mid descending aorta through median sternotomy alone, avoiding left thoracotomy. Karck and Kamiya ${ }^{15}$ reviewed the frozen elephant trunk in 215 patients from 8 reports, and the mortality rate of this procedure including acute aortic dissection was $15(7.0 \%)$ of 215 . They concluded that the frozen elephant trunk technique might become the next standard treatment for extended aortic aneurysm instead of its conventional variant. We had no operative mortality and only 1 in-hospital mortality in this series that was limited to elective arteriosclerotic aneurysm. Moreover, we had good results in that the arteriosclerotic aneurysm excluded by the stent graft shrank in $68 \%$ and was obliterated in $18 \%$ after the frozen elephant trunk procedure according to the long-term CT observation.

On the other hand, the frozen elephant trunk had several disadvantages such as risk of distal endoleak and spinal cord injury compared with the conventional replacement.

TABLE 4. Size of aneurysm (computed tomographic data)

\begin{tabular}{lc}
\hline Follow up $>\mathbf{6}$ mo & $\mathbf{N}=\mathbf{5 0}$ \\
\hline Increase (\%) & $1(2.0)$ \\
No change (\%) & $6(12.0)$ \\
Shrinkage (\%) & $34(68.0)$ \\
Obliteration (\%) & $9(18.0)$ \\
Endoleak (\%)* & $1(2.0)$ \\
\hline
\end{tabular}

*In a patient who had an increase in aortic size on the aneurysm, a distal endoleak occurred 42 months after the operation; he was listed for elective thoracic endovascular aortic repair.
We experienced 1 case of late endoleak 42 months after the operation. This case occurred as a result of migration toward the aortic arch with a spindle-shaped aneurysm whose diameter was $65 \mathrm{~mm}$. We should warn that the frozen elephant trunk for spindle-shaped aneurysm with large diameter has the risk of distal endoleak during long-term follow-up. The stent graft design for the frozen elephant trunk should be improved to avoid migration during long-term follow-up.

Spinal cord ischemia is known to a devastating complication of the frozen elephant trunk..$^{7,9,14,15}$ The incidence of paraplegia after implantation of the frozen elephant trunk is $3.3 \%$ according to a review by Karck and Kamiya ${ }^{15}$ Spinal cord injury after the frozen elephant trunk was associated with multiple factors, such as the thoracic vertebral level, where the distal end of the frozen elephant trunk was deployed, a history of downstream aortic surgery, intraoperative hypotension, and each patient's individual pathologic condition (aneurysm or acute dissection). Our series showed $2(3.4 \%)$ cases of spinal cord injury because of the deep positioning of the stent graft, the simple clamping of the left subclavian artery without perfusion, and the previous surgery of the abdominal aortic aneurysm. Cerebrospinal fluid drainage was performed before the operation in elective cases with a history of aortic repair in the thoracoabdominal or abdominal aorta or when the pathologic condition required stent graft delivery lower than Th9 after January 2002. No spinal cord ischemia has been observed since then.

\section{Limitations of this Study}

This is a retrospective cohort study. A prospective study would be required to elucidate the precise advantage of the frozen elephant trunk technique.

\section{CONCLUSIONS}

The frozen elephant trunk for extended arteriosclerotic aneurysm from the arch to the descending aorta had long-term durability and efficacy of this one-stage procedure from our 11 years of experience in 58 consecutive patients. We concluded that the frozen elephant trunk technique might become the alternative treatment for extended aortic aneurysm.

\section{References}

1. Kouchoukos NT, Mauney MC, Masetti P, Castner CF. Optimization of aortic arch replacement with a one-stage approach. Ann Thorac Surg. 2007;83:S811-4.

2. Doss M, Woehleke T, Wood JP, Martens S, Greinecker GW, Moritz A. The clamshell approach for the treatment of extensive thoracic aortic disease. $J$ Thorac Cardiovasc Surg. 2003;126:814-7.

3. Safi HJ, Miller CC III, Estrera AL, Villa MA, Goodrick JS, Porat E, et al. Optimization of aortic arch replacement: two-stage approach. Ann Thorac Surg. 2007;83:S815-8.

4. LeMaire SA, Carter SA, Coselli JS. The elephant trunk technique for staged repair of complex aneurysms of the entire thoracic aorta. Ann Thorac Surg. 2006;81: 1561-9.

5. Svensson LG, Kim KH, Blackstone EH, Alster JM, McCarthy PM, Greenberg RK, et al. Elephant trunk procedure: newer indications and uses. Ann Thorac Surg. 2004;78:109-16. 
6. Kato M, Ohnishi K, Kaneko M, Ueda T, Kishi D, Mizushima T, et al. New graftimplanting method for thoracic aortic aneurysm or dissection with a stented graft. Circulation. 1996;94(Suppl). II188-93.

7. Baraki H, Hagl C, Khaladj N, Kallenbach K, Weidemann J, Haverich A, et al. The frozen elephant trunk technique for treatment of thoracic aortic aneurysms. Ann Thorac Surg. 2007;83:S819-23.

8. Liu ZG, Sun LZ, Chang Q, Zhu JM, Dong C, Yu CT, et al. Should the "elephant trunk" be skeletonized? Total arch replacement combined with stented elephant trunk implantation for Stanford type A aortic dissection. J Thorac Cardiovasc Surg. 2006;131:107-13.

9. Uchida N, Ishihara H, Sakashita M, Kanou M, Sumiyoshi T. Repair of the thoracic aorta by transaortic stent grafting (open stenting). Ann Thorac Surg. 2002;73: 444-9.

10. Flores J, Kunihara T, Shiiya N, Yoshimoto K, Matsuzaki K, Yasuda K. Extensive deployment of the stented elephant trunk is associated with an increased risk of spinal cord injury. J Thorac Cardiovasc Surg. 2006;131:336-42.
11. Antona C, Vanelli P, Petulla M, Gelpi G, Danna P, Lemma M, et al. Hybrid technique for total arch repair: aortic neck reshaping for endovascular-graft fixation. Ann Thorac Surg. 2007;83:1158-61.

12. Melissano G, Civilini E, Bertoglio L, Calliari F, Setacci F, Calori G, et al. Results of endografting of the aortic arch in different landing zones. Eur J Vasc Endovasc Surg. 2007;33:561-6.

13. Uchida N, Ishihara H, Shibamura H, Kyo Y, Ozawa M. Midterm results of extensive primary repair of the thoracic aorta by means of total arch replacement with open stent graft placement for an acute type A aortic dissection. J Thorac Cardiovasc Surg. 2006;131:862-7.

14. Shimamura K, Kuratani T, Matsumiya G, Kato M, Shirakawa Y, Takano H, et al Long-term results of the open stent-grafting technique for extended aortic arch disease. J Thorac Cardiovasc Surg. 2008;135:1261-9.

15. Karck M, Kamiya H. Progress of the treatment for extended aortic aneurysms; is the frozen elephant trunk technique the next standard in the treatment of complex aortic disease including the arch? Eur J Cardiothorac Surg. 2008;33:1007-13. 